\title{
1 Die Vergegenwärtigung des Lebens und die Evolution des Bewusstseins
}

\author{
Joachim Galuska
}

Moderne Spiritualität ist letztendlich die Entfaltung des eigenen Bewusstseins. So bezog sich der Fokus meiner persönlichen Suche auf die Arbeit an der Ausdehnung meines Bewusstseins. Die Anwendung dieser Bewusstseinsformen und Bewusstseinszustände, z.B. in der Psychotherapie, in der Unternehmensführung oder in meiner Lebensführung, erschienen mir wie Früchte auf diesem Weg. So begann ich immer mehr in meinem Leben aufzuwachen und das Leben zu vergegenwärtigen, das ich gerade lebte. Ich begann, mich an den Platz in meinem Leben zu stellen, an dem ich eben gerade stand, mich in die Mitte des Lebens hineinzustellen als der, der ich eben war oder bin, mit allen meinen Eigenschaften, meiner Geschichte, meinem Sein, meinem Bewusstsein, meinen Bezügen und meiner Welt. Ich spürte die Bedeutung der Worte von Albert Schweitzer (1974): „Ich bin Leben, das Leben will, inmitten von Leben, das Leben will." Ich begann das Leben zu vergegenwärtigen, so wie es gerade geschieht, die Momente meines Lebens zu vergegenwärtigen in ihrer Ganzheit und ihrer lebendigen Präsenz. Ich spürte den Albert Schweitzerschen Satz noch weiter vereinfacht: „Ich bin Leben, das lebt, inmitten von Leben, das lebt.“ Mein persönliches und individuelles Leben als Teil eines umfassenderen Lebensprozesses zu erkennen, ließ mich mein Leben und mehr noch das Leben selbst erforschen. Ich begann, inmitten des Lebens das Leben selbst zu spüren in seiner Lebendigkeit, in seiner enormen Präsenz, in seinem Strömen, dem Strömen unseres Lebensstromes, in seinem Fließen, dem Fließen des Flusses unseres Lebens, in seiner Ursprünglichkeit, pur, pures Leben. Ich begann zu spüren, was für eine Freude es ist, das Leben in seiner Essenz zu spüren, die Lebensfreude in ihrer Ursprünglichkeit zu empfinden, was für ein Clück es ist zu leben, gerade zu leben, diesen Quell dieses Stromes des Lebens zu spüren und ein Teil davon zu sein. Und ich begann, den Wert 
dieses Lebens zu spüren, wie großartig es ist, wie intelligent, und wie es sich entfaltet. Dass ich und wir nicht nur daran teilhaben können, sondern dies auch noch vergegenwärtigen können, erkennen können, dies erleben dürfen am Leben zu sein, zu leben, empfinde ich als eine große Gnade, das größte Geschenk, das jeder von uns erhalten hat und in sich trägt, solange er lebt.

Auf meinem Weg habe ich mich immer wieder gefragt, was eigentlich fundamentaler ist: Das Bewusstsein oder das Leben?

Ist die zentrale evolutionäre Bewegung die des Bewusstseins, das sich selbst vergegenwärtigt als Ausdruck einer umfassenderen Intelligenz, die sich als dieses Universum manifestiert, als diese Evolution, die irgendwann einmal Leben hervorbringt und Nervensysteme, die erwachen und sich selbst erkennen als Teil dieser umfassenderen Intelligenz?

Oder ist der evolutionäre Prozess in sich selbst ein lebendiger Ausdruck dieser umfassenderen Intelligenz, eine lebendige Evolution, die sich im Zuge ihrer eigenen Bewusstwerdung und Vergegenwärtigung spürt und in voller Bewusstheit weiterentwickelt?

Mit zunehmendem Alter neige ich zu der zweiten Variante, das Leben zu begreifen als ein sich selbst aufklärender Prozess, als ein Geschehen, das sich seiner selbst bewusst wird und sich selbst zunehmend erkennt und vergegenwärtigt in seiner Art und Weise, in seinen Grundstrukturen, seinen innersten und äußersten Bewegungen, seinem evolutionären Potenzial, seiner eigenen Intelligenz. Der Fokus der Erforschung des Lebens liegt dann nicht mehr primär in der Vertiefung und Erweiterung des Bewusstseins, der inneren Loslösung aus den fixierenden Strukturen und Mustern und der Konzentration auf eine transzendente Wirklichkeit, sondern er bezieht sich auf das Innerste des Lebens selbst, auf eine Vertiefung und Erweiterung unseres Lebens.

\section{Der Weg des Lebens}

Was geschieht, wenn wir inmitten unseres Lebens aufwachen, inmitten dieses Momentes, und ihm nichts entgegensetzen? Dann spüren wir unsere lebendige Präsenz, unsere lebendige Anwesenheit.

Was spüren wir, wenn wir inmitten dieses Momentes, in dieser Präsenz ganz offen sind und in völliger Offenheit verweilen? Dann spüren wir die Fülle dieses Momentes, die Fülle dieser gesamten Erfahrung.

Die innere Leere unserer schwebenden Offenheit lässt uns die Fülle spüren unserer Sinneserfahrungen, unserer Empfindungen und Gefühle, unseres Bewusstseins.

Und wie ist es, uns an diese Fülle hinzugeben, uns ganz ausfüllen und erfüllen zu lassen von der Fülle des Lebens, uns ergreifen zu lassen von seiner Intensität, seinen Lebensenergien, dem Strömen, das von innen heraus diese Lebenserfahrung hervorbringt und weiter entfaltet? Es ist zumindest belebend, wahrscheinlich auf eine innerste Weise ästhetisch, einfach schön und lustvoll, letztendlich sogar ekstatisch. Das Leben von innen her in seiner überfließenden Fülle und Schönheit zu spüren ist letztendlich Ekstase, Teilhabe an der Freude des Lebens an sich selbst, am Geschmack des sich entfaltenden Lebensstromes. Vieles könnte und sollte man vielleicht auch an dieser Stelle sagen über Lebensfreude und Lebenslust, über Schönheit und Ästhetik. Sie wecken jedenfalls unser Herz und lassen uns dem Leben zuwenden, das Leben annehmen und lieben. Unser Herz zu öffnen und uns verbunden sein zu lassen mit den Menschen, der 
Natur, der Welt und dem Göttlichen und Unbekannten, erleichtert es enorm, auch das Leben anzunehmen, sich mit ihm verbunden sein zu lassen und es dann zunehmend in seinem Innersten zu spüren und sich von ihm ergreifen und führen zu lassen.

Mit dem Leben verbunden zu sein bedeutet, es ganz anzunehmen,

- nicht nur in seiner Schönheit, sondern auch in seinem Schrecken, wie Rilke sagt,

- nicht nur in seiner Leichtigkeit, sondern auch in seinem Ernste, wie Rilke ebenfalls sagt,

- nicht nur in seiner Lebensfreude, sondern auch in seinem Schmerz und seinem Leid,

- nicht nur in seiner Tiefe, sondern auch in seiner Oberfläche,

- nicht nur in seiner individuellen Entfaltung, sondern auch in seiner kollektiven Verbundenheit.

Den Weg des Lebens zu gehen bedeutet auch, das eigene persönliche Leben anzunehmen, mich anzunehmen als Ausdruck meines Lebens. Und das ist vielleicht das schwierigste: mich und mein Leben vollkommen zu akzeptieren und anzunehmen, so wie es ist und so wie ich bin, ohne Ablehnung, ohne Widerstand. Es bedeutet nicht, alles zunächst gut zu heißen oder schön zu finden, sondern eher es hinzunehmen, zunächst einmal sein zu lassen, zu spüren wie es ist, in allen seinen Licht- und Schattenseiten. Und es ist eben ein Sich-mitten-hineinstellen in dieses Leben, ein Aufwachen in diesem Fluss meines Lebens und ein Vergegenwärtigen, wie es sich anfühlt und wie es ist, in dieser Zeit, auf diesem Planeten, in dieser Familie, in dieser Kultur geboren zu sein und als Mensch in dieser Form zu leben. All dies ist Ausdruck des Lebens und gehört zu meinem persönlichen und individuellen Leben. Und wenn ich es spüre und erkenne und zu mir nehme, dann finde ich mich selbst darin und entdecke, dass ich mich darin bewegen kann. Und ich spüre, welches ungeheure Potenzial in diesem Leben als Mensch liegt, welche Möglichkeiten ich besitze, welche Kompetenzen, mein Leben zu gestalten und die Welt zu verändern. Wenn ich mein Leben ganz annehme, steht mir auch meine gesamte Lebensenergie zur Verfügung, meine gesamte Schaffenskraft, mein gesamtes schöpferisches Potenzial. Ich kann spüren, wie ich in meinem Leben wirken kann, verändern kann, gestalten kann, wie ich dabei an Grenzen stoße, aber auch Grenzen überwinde. Ich kann diesen kreativen Prozess des Lebens von innen her wahrnehmen und in meinem Leben zum Ausdruck kommen lassen. Und wenn ich mein Leben lieben lerne und mich mit ihm verbunden fühle, dann spüre ich diese immense und unmittelbare Verantwortlichkeit, die ich mir und meinem Leben gegenüber besitze, mein Leben auf eine lebenswerte Weise zu gestalten.

Den Weg des Lebens zu gehen heißt, das Leben in allen seinen Facetten zu durchdringen und zu verinnerlichen, das Leben von innen her zu spüren und zu leben. Unsere Bewusstseinsentwicklung kann dazu dienen, das Leben immer tiefer und weiter zu verstehen und zu durchdringen. Je höher entwickelt die Bewusstseinsstruktur, umso tiefer und umfassender ist das Verständnis des Lebens, umso mehr geschieht eine Verbundenheit und schließlich ein Einswerden mit dem Lebensstrom und dann auch mit der Intelligenz, die das Leben so sein und leben lässt, wie es eben lebt, die es konfiguriert und strukturiert. Und je mehr dieses Verinnerlichen geschieht, dieses vergegenwärtigte Leben zu leben, umso größer und tiefer ist die Freiheit und umso umfassender ist das Potenzial es zu entfalten. Es braucht den Weg des Bewusstseins, um Seele, Geist und kosmische Intelligenz zu erspüren, und es braucht eine Öffnung für das Leben, das eh da ist, pulsiert, strömt und geschieht, um ein beseeltes und intelligentes Leben zu führen. 
Leben will leben, wie Schweitzer sagt. Leben will erlebt werden. Leben will sich entfalten und erfüllen. Leben will so voll wie möglich leben. Aber wenn wir das Wollen aus all dem heraus lassen, dann tut es das einfach, und wir gestalten unser Leben mit zunehmender Vergegenwärtigung unseres Lebens, mit zunehmendem Spüren unseres Lebens, im Einklang mit dem Leben.

\section{Der kollektive Lebensprozess}

Den Weg des Lebens zu gehen bedeutet, nicht nur das eigene persönliche Leben anzunehmen, sondern auch zu spüren wie es ist, Teil einer lebendigen Gemeinschaft von Lebewesen zu sein, die eben gerade leben. In dieser offenen Präsenz zu schweben, bedeutet zu spüren, wie es ist, inmitten von Leben zu sein, wie es Albert Schweitzer tat. Das Leben der Lebewesen um mich herum zu spüren und zu realisieren, wie das Leben sich in allen Lebewesen ereignet, lässt mich teilhaben an der Fülle der lebendigen Entfaltungen dieser Evolution. Sie eröffnet mir einen ungeheuren Reichtum an Erfahrungen und eine unermessliche Vielfalt und Tiefe von Begegnungsmöglichkeiten. Sie weckt aber auch mein Mitgefühl für all das Schmerzliche, Leidvolle, Verirrte und Verwirrte. Und sie lässt mich meine Mitverantwortlichkeit spüren für diese Gemeinschaft, zu der ich gehöre, allein deshalb, weil ich gerade in und mit ihr lebe. Sie lässt mich meine Teilhabe an allem Leben und letztlich an der gesamten Evolution spüren, denn sie ist ja auch Ausdruck des Lebens. Sie lässt mich diese kollektive Qualität, diese Gemeinschaftlichkeit, diese Zusammengehörigkeit unmittelbar spüren. Nicht nur ich lebe, sondern wir leben und ich gehöre dazu! Wir leben, wir leben als Familie, wir leben als Arbeitsgemeinschaft, wir leben als Gesellschaft, wir leben als Mensch heit, wir leben als Gemeinschaft aller Lebewesen, wir leben als Natur, wir leben als Kosmos. Wir sind diese kosmische und universelle Intelligenz, die eben so lebt und sich entfaltet.

Es ist letztendlich kein Verlust, sein Leben hinzugeben an diesen kollektiven Lebensprozess, sein Leben in den Dienst der Gemeinschaft zu stellen, zu der man gehört. Das Leben gibt uns frei, unser Leben individuell zu entfalten, aber es bereichert es enorm, unser Leben gemeinsam zu entfalten. Wenn wir danach fragen, was uns Menschen am meisten erfüllt, sind es Partnerschaft und Familie und eine sinnvolle Arbeit, die ja immer etwas für andere Menschen tut. Sowohl aus der Sicht der Bewusstseinsentwicklung als auch aus der Sicht der gemeinsamen Lebensgestaltung ist die Mitverantwortung und die Mitwirkung an unserer Kultur - also unserem gemeinsamen Bewusstseins- und Lebensfeld - eigentlich eine selbstverständliche und natürliche Haltung. Bewusstseinserforschung als Entwicklungsweg führt aber etwas eher zur individuellen Entfaltung, zur individuellen inneren Befreiung und zur individuellen Verwirklichung und Anwendung der gewonnenen Einsichten. Lebenserforschung führt eher zur Lebensbejahung und zur „Ehrfurcht vor dem Leben“, wie Albert Schweitzer sagt, und das meint sowohl mein persönliches Leben als auch alles Leben.

Die eigentliche Lebenskunst besteht nicht nur darin, mein eigenes Leben kreativ und erfüllt zu leben, sondern es im Einklang mit allem Leben zu gestalten. Um es einmal poetisch auszudrücken: „Meine Melodie in der Symphonie des Lebens zu spielen.“ Mein Leben und unser Leben gehören untrennbar zusammen. Jeder von uns erleidet sein eigenes Leben und unser gemeinsames Leben. Jeder von uns genießt sein eigenes Leben und unser gemeinsames Leben. Und wir können gemeinsam großartige Lebenskunstwerke schaffen, großartige Symphonien miteinander improvisieren, wenn wir nicht so viel Lärm machen und mehr auf die Töne und Klänge der anderen lauschen. 
Aber was geschieht, wenn wir dies vergegenwärtigen, dass wir eben gerade gemeinsam unser Leben gestalten, nicht nur unser Bewusstsein bilden, sondern hier und jetzt leben?

Ich halte dies für pure kollektive Spiritualität. Mein spirituelles Verständnis hat mich letztendlich zum Leben geführt. Auch ich bin den Weg des Bewusstseins gegangen, zunächst zu versuchen, meinen Geist zu befreien von allen Verhaftungen in der Welt und offen zu werden für die Größe und Unmittelbarkeit des Absoluten und Unbekannten. Aber ich habe mehr und mehr gesehen, dass dies nur Bedeutung hat, wenn es mein Leben verändert und erfüllt, nicht wenn es mich von meinem Leben befreit. Meine Bewusstwerdung hat mich mein Leben spüren lassen und das größere Leben, das uns alle hervorbringt. Sie hat den Blickwinkel meiner Spiritualität weggeführt von dem Höheren, Visionären und Zukünftigen hin zum Unmittelbaren, Gegenwärtigen und Lebendigen. Eine Spiritualität des Lebens befreit nicht vom Leben, sondern sie befreit zum Leben, das aufwacht und sich seiner selbst vergegenwärtigt und sich seines Daseins erfreut, das spielt und tanzt. Und es forscht und sucht:

Das Leben sucht Glück und es findet Glück.

Es sucht Frieden und es findet den Frieden.

Es sucht Begegnung und es findet andere.

Es sucht Stille und findet Stille.

Es sucht Bewegung und es findet Bewegung.

Es sucht Liebe und es findet die Liebe.

Es sucht Großes und es findet Großes.

Es sucht das, was es überschreitet und es findet das, was es überschreitet.

Das Leben verliert sich und vergisst sich und schläft ein und wacht wieder auf.

Es wird krank und leidet und manchmal heilt es und manchmal nicht.

Es wird geboren und wächst und stirbt - irgendwann.

Es lebt als Ich, als Wir und als alles, was lebt.

\section{Evolution des Bewusstseins}

In der Entfaltung unseres Bewusstseins wacht Gott auf, könnte man sagen, und er erkennt und erfährt sich selbst - in uns - als wir - als Du und Ich. Unser scheinbar kleines Leben ist das große Leben, wenn wir es von innen her in der Tiefe durchdrungen haben. Und dieses Leben entfaltet sich in jedem von uns, und wir spüren diese Dynamik, dieses Wachsen und Vergehen, diese innere Bewegung, diese Entwicklung. Und wir spüren, wie es ist, aufzuwachen, zu leben, sich zu entfalten, sich zu entwickeln. Wir sind diese Entwicklung. Als Mensch vergegenwärtigt sich diese ungeheure Intelligenz ihrer eigenen Entwicklung, ihrer eigenen Evolution als diese Evolution. Wir haben Teil an diesem gewaltigen intelligenten evolutionären Prozess, zu dem wir gehören, und wir spüren, wie dieser Prozess sich aus sich selbst heraus entfaltet. Wir spüren, wie dieser Prozess sich selbst aufklärt, sich seiner selbst bewusst wird als Mensch und sich in dieser Bewusstwerdung weiter entfaltet und weiter gestaltet. Wir spüren diese sich selbst entfaltende Lebendigkeit und Kreativität dieses evolutionären Prozesses. Wir spüren in uns eine schöpferische Kraft als Qualität unseres eigenen Lebens. Das Schöne am Menschsein ist, nicht nur das Leben zu erleben, sondern sich in diesem lebendigen Prozess bewegen zu können, das eigene Leben zu gestalten, zu verändern und damit diesen gesamten evolutionären Strom 
mit zu gestalten, mit zu verändern. Es ist wunderschön zu spüren, zu fühlen, zu realisieren, zu vergegenwärtigen, das Leben in voller Bewusstheit zu erleben. Aber es ist ebenso wunderschön, sich bewegen zu können, wirken zu können, kreativ sein $\mathrm{zu}$ können, Neues zu finden oder zu erfinden und zu verwirklichen und beteiligt $\mathrm{zu}$ sein an dieser gemeinsamen Konstruktion unserer Wirklichkeit, an dieser gemeinsamen Gestaltung unseres Lebens, an dieser gemeinsamen Weiterentwicklung dieser Evolution. Es ist wunderschön, an diesem großen Entfaltungsprozess des Lebens beteiligt zu sein - ja, ein Teil von diesem Prozess zu sein und ihn zusammen mit allem anderen Lebendigen mitzugestalten. Und es ist beeindruckend, die Intelligenz dieses Prozesses zu spüren, die dies entwirft, strukturiert, konfiguriert. Diese Intelligenz überschreitet die gegenwärtige Reichweite unseres Bewusstseins. Wir können sie nicht erfassen, weil die Strukturen unseres Geistes lediglich oberflächliche Spiegelungen von ihr darstellen. Aber sie lässt sich spüren. Für mich fühlt sie sich an wie ein funkelnder in sich selbst freier, intelligenter Raum, der sich jedem geistigen Erkennen gegenwärtig noch entzieht. Aber letztendlich bin ich und ist jeder von uns nichts anderes als diese lebendige Intelligenz, aufgewacht und vergegenwärtigt in ihrem eigenen evolutionären Prozess. Und dieser evolutionäre Prozess selbst ist intelligent, voller Potenzial, bewegt sich in einer Art freien Entfaltung, eröffnet Perspektiven und Dimensionen, erfüllt und transzendiert unser Leben.

Wenn wir die Entwicklung unseres Bewusstseins auf diese Weise erkennen können, sind wir nicht länger Produkte der Evolution, ist unser Bewusstsein nicht länger Ergebnis einer evolutionären Entwicklung von Nervensystemen, sind wir nicht länger Erleidende unseres Lebens und durch die schicksalhaften Wechselfälle Aufgeweckte, sondern wir sind angekommen, da wo wir sind. Wir sind aufgestanden an der Stelle, an der wir stehen. Wir nehmen unser Leben und das aller Lebewesen, mit denen wir verbunden sind, in Würde an. Wir nehmen die Verantwortung an, die es bedeutet, Mitgestalter dieses Lebens und dieses evolutionären Prozesses zu sein. Wir erkennen, dass wir uns dem nicht entziehen können, dass jeder von uns darin notwendig ist, eine Stimme besitzt, Einfluss nimmt. Wir erkennen, dass jeder von uns darin gebraucht wird, wie Martin Buber (1922) sagt:
„Dass du Cott brauchst, mehr als alles, weißt du allzeit in deinem Herzen; aber nicht auch, dass Gott dich braucht, in der Fülle seiner Ewigkeit dich? Wie gäbe es den Menschen, wenn Gott ihn nicht brauchte, und wie gäbe es Dich? Dubrauchst Gott, um zu sein, und Cott braucht dich - zu eben dem, was der Sinn deines Lebens ist ... Die Welt ist nicht göttliches Spiel, sie ist göttliches Schicksal. Dass es die Welt, dass es den Menschen, dass es die menschliche Per- son, dich und mich gibt, hat göttlichen Sinn. Schöpfung - sie geschieht an uns, sie glüht sich uns ein, glüht uns um, wir zittern und vergehn, wir unterwerfen uns. Schöpfung - wir nehmen an ihr teil, wir begegnen dem Schaffenden, reichen uns ihm hin, Helfer und Gefährten."

Aber was ist, wenn Gott und ich eins sind, wenn das göttliche Schicksal unser Leben ist, wenn wir die Schöpfung, die sich uns einglüht nicht nur erleiden, sondern sie gestalten, entwickeln, entfalten. Dann gibt es niemanden anderes, den wir verantwortlich machen können, keinen Gott, kein Absolutes, keine Intelligenz außerhalb oder irgendwo jenseits von uns, sondern wir sind es selbst. Es ist unser eigener Lebensprozess, unsere eigene Evolution. Und ich meine dies nicht als überschätzte persönliche Macht, sondern es geht darum, das eigene persönliche Leben in den Dienst zu stellen unserer Mitverantwortung für die Evolution, und diese auf eine menschenwürdige Weise, intelligent und weise, sowohl in Demut als auch in Frei- 
heit weiterzuentwickeln. John Stuart (2012), ein australischer Evolutionsforscher, nennt uns „evolutionäre Aktivisten“, „absichtsvolle Evolutionäre“:

„Evolutionäre Aktivisten nutzen die Gerichtetheit der Evolution, um herauszufinden, was sie tun müssen, um diese weiter voranzubringen. Im sozialen Bereich ist der nächste große Schritt der menschlichen Evolution das Hervorbringen einer geeinten und nachhaltigen globalen Gesellschaft. Psychologisch betrachtet ist es die Befreiung unseres Verhaltens vom Diktat der biologischen und kulturellen Vergangenheit, damit wir das, was für die zukünftige evolutionäre Möglichkeit nötig ist, tun können."

„Wenn der Übergang zur bewussten Evolution vollzogen ist, wird die Evolution auf der Erde bewusst und intelligent von statten gehen."

Evolutionäres Bewusstsein bedeutet für mich, das jeweils geistig Erkannte auch erfahren zu wollen und dann anderen weiterzugeben und ihnen erfahrbar zu machen, sodass die ganze Entwicklung damit stabiler und durchdrungener wird.

„Durch die Menschen wird sich der evolutionäre Prozess seiner selbst bewusst geworden sein und die Kapazität haben, sich selbst absichtsvoll und bewusst weiterzuentwickeln." (Stuart 2011).

Darauf scheint mir der Sinn des bewusst gewordenen, des vergegenwärtigten Lebens hinauszulaufen:

Mit Hilfe meiner gesamten Intelligenz, meiner intuitiven Kompetenz, verankert in meiner Seele und meiner lebendigen Offenheit, verbunden mit der Welt und diesem evolutionären Prozess, mein Leben und unser Leben zu gestalten und bewusst weiterzuentwickeln. Und dies ist keine moralische Forderung, sondern ein tiefes inneres Gespür, eigentlich sogar eine Gnade, ein großes Geschenk, ein solches Leben gegenwärtig leben zu dürfen. Und wenn ich mich frage, was diese Vergegenwärtigung für mein gegenwärtiges Leben bedeutet, für das, was ich konkret und praktisch tue, dann stellt sich diese Frage auch Gott oder diese universelle Intelligenz, denn letztlich ist da ja kein Unterschied. Also was wollen wir tun und zwar weil wir es wirklich wollen, weil wir diese Verantwortlichkeit spüren, weil wir diese Liebe zum Leben spüren, weil wir es gerne tun wollen oder weil wir Mitgefühl besitzen, weil etwas uns berührt oder empört oder weil es Freude macht und uns begeistert?

An dieser Stelle inmitten des Lebens, inmitten dieses evolutionären Prozesses sind wir letztlich frei. Wir sind zwar verbunden mit all dem, wir werden von all dem hervorgebracht und genau an die Stelle gestellt, an der wir eben stehen und leben. Aber wenn wir sie vergegenwärtigen, dann spüren wir eben auch die vollkommene und tiefe innere Freiheit, die in unserem Innersten liegt und die bei allen vorgegebenen Strukturen eben auch unser eigenes Leben und das dieser gesamten Evolution in sich trägt. Dann spüren wir wie es ist, unbestimmt und offen zu sein, frei für unsere Kreativität, frei für etwas völlig Neues, nicht letztlich vorhersagbar. Und das ist das Schöne, dass unser Wollen letztlich freiwillig ist. Was bin ich bereit, freiwillig zu tun, freiwillig einzubringen, weil es mir am Herzen liegt, nicht weil ich mir persönlichen Profit davon verspreche? Oder wieviel persönlicher Profit ist wirklich erforderlich in all dem, was ich tue in meinem Leben, in all dem, was ich hineingebe ins Leben, weil ich es bereichern will, aufklären will, weiterentwickeln will, erlösen will und nicht weil ich unbedingt muss, sondern weil es mir am Herzen liegt, weil mir etwas weh tut oder mich etwas wirklich inspiriert? 
Ich bin überzeugt davon, dass unser Leben reicher und erfüllter ist, wenn wir uns ihm nicht entziehen, wenn wir uns nicht nur zurückziehen in die Abgeschiedenheit und Stille im Inneren vom Äußeren, wenn wir uns nicht nur um uns selbst drehen und nicht nur nehmen, was wir kriegen können, sondern wenn wir bereit sind, uns ganz hineinzugeben, uns „nicht vorzuenthalten“, wie Martin Buber (1919) sagt:

„Durchbrich deine Schalen, werde unmittelbar, rühre, Mensch, die Menschen an.“

Ich persönlich halte dies nicht für eine Pflicht oder einen Imperativ, sondern für eine natürliche Bewegung des erwachten und vergegenwärtigten Lebens, dies freiwillig zu wollen. Es geschieht aus der Verbundenheit mit dem evolutionären Prozess. So wie viele Menschen früherer Zeiten und auch gegenwärtig mein Bewusstsein und mein Leben bereichern, möchte ich auch meinen Teil zu ihrem Leben und dem der folgenden Generationen beitragen. Vielleicht erleiden die Menschen der Zukunft dann nicht nur unser Erbe, sondern freuen sich auch daran. Doch natürlich will das Leben auch spielen und lachen und tanzen. Und auch das bereichert unser gemeinsames Leben und erfüllt und steckt an und lässt uns unser Leben feiern. Aber auch dafür braucht es weiterentwickelte Arten und Weisen, die der Größe unseres Lebens und unserer inneren Freiheit entsprechen.

\section{Eine neue abendländische Spiritualität}

So wird eine neue abendländische Spiritualität die bewusste Evolution in ihren Mittelpunkt stellen. Sie wird zwar ansetzen an der Arbeit am eigenen Bewusstsein, an der Öffnung und Vertiefung unseres „Weltinnenraumes“. Und dies erfordert unser Erwachen, unser Bewusstwerden, die Relativierung unserer Identifikationen und unserer Konstruktionen, die Suche nach dem, was uns überschreitet. Es ist wie ein Aufschwingen zu einem größeren Überblick, zu einer größeren geistig-spirituellen Weite und erfordert eine gewisse Mühe und Disziplin, auch wenn es auf ein „müheloses Bemühen“ hinausläuft. Eine neue abendländische Spiritualität wird aber basieren auf der Vergegenwärtigung des Lebens. Sich mit dem eigenen Leben verbunden zu fühlen, so wie es ist, sich an die Stelle im Leben zu stellen, an der man eben steht, das Leben anzunehmen, das man eben lebt, ist leichter und versöhnlicher. Wenn wir uns dem Leben anvertrauen erfahren wir, wie das Leben von alleine geschieht, als eine aus sich selbst herausströmende Quelle, die auch unser Leben hervorbringt. Es ist, als ob uns das Leben in den Arm nimmt und wieder ausatmet, durch unsere Sinne und unseren Geist in die Welt hinaus sendet, wie Rilke in seinem Gedicht sagt: „Von deinen Sinnen hinausgesandt, geh bis an deiner Sehnsucht Rand ..." Eine neue abendländische Spiritualität wird nicht die Sinne ablehnen, sondern Fülle, Freude und Ekstase genauso würdigen, wie Berührung und Verwandlung von Leid und Schmerz. Eine neue abendländische Spiritualität wird also neben der Arbeit an der Erforschung des eigenen Bewusstseins genauso sehr die Durchdringung und Entfaltung des eigenen Lebens betonen und schließlich eine Spiritualität sein, in der wir unseren Beitrag zur gemeinsamen Gestaltung der Welt leisten. Sie wird eine Spiritualität sein, in der unser Leben immer mehr durchdrungen wird vom Größeren, Unbekannten und Unbegreiflichen und schließlich ein vergegenwärtigtes Geschehen einer intelligenten Evolution wird. 
1 Die Vergegenwärtigung des Lebens und die Evolution des Bewusstseins

\section{Literatur}

Buber M (1919) Was ist zu tun? In: Hinweise, Gesammelte Essays. Manesse Verlag 1953

Buber M (1922) Ich und Du. Reclam Verlag Stuttgart

Schweitzer A (1974) Gesammelte Werke. In: Grabs R (Hrsg.) Bd. 1: Aus meinem Leben und Denken; Aus meiner Kindheit und Jugendzeit; Zwischen Wasser und Urwald; Briefe aus Lambarene 1924-1927. 169. Beck, München

Stuart I (2012) Die Absicht des Kosmos. In: EnlightenNext Impulse 3/2012, 24-27

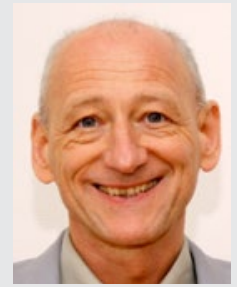

\section{Dr. med. Joachim Galuska}

Facharzt für Psychosomatische Medizin und für Psychiatrie und Psychotherapie, Geschäftsführer und Ärztlicher Direktor der Heiligenfeld Kliniken in Bad Kissingen und Waldmünchen. Herausgeber u.a. der Bücher: Die Kunst des Wirtschaftens (2010), Psychotherapie und Bewusstsein (2005), Gründer der Zeitschrift Bewusstseinswissenschaften und der Stiftung Bewusstseinswissenschaften. 
